\title{
SIMULTANEOUS JOINT SCALING TEST FOR SEVERAL CORRELATED TRAITS*
}

\author{
M. SINGH \\ Statistics Unit, International Crops Research Institute for the Semi Arid Tropics, Patancheru, \\ Andhra Pradesh 502 324, India
}

Received 23.iii.83

\section{SUMMARY}

\begin{abstract}
For generation mean analysis a simultaneous joint scaling test with several correlated traits is proposed. Scaling test for individual traits were compared with this test and in general were not independent and are relatively less efficient for the correlated traits situation. The two and three traits cases have been illustrated with real data.
\end{abstract}

\section{INTRODUCTION}

Generation mean analysis is often used to estimate components of mean (additive and dominance effects and interaction and heterosis) of individual traits. A joint scaling test proposed by Cavalli (1952) and described in Mather and Jinks (1971) is generally used for testing the appropriateness of the mathematical model before estimating or testing the significance of the components of means. A number of papers (e.g., Pathak and Singh (1970), Law et al. (1978)) present generation mean analyses for several traits considered individually. However, the traits may be significantly correlated, perhaps due to linkage, so that inferences from scaling tests applied to individual traits are dependent. A simultaneous scaling test is proposed which tests genetic models for several traits and estimates various components while allowing for correlations among them. Estimators of the mean components from the simultaneous scaling test will be more efficient than those from scaling tests applied on individual traits. However, as far as is known, the improvement in efficiency is relatively small.

A simultaneous scaling test for any number of correlated traits is presented in section 2 . The two traits case and some statistical consideration of the two tests are given in section 3 and illustrated in section 4 using the referee's analysis for two and three traits data of his colleagues.

\section{Simultaneous JoINT SCAling TEST}

Consider the situation where a plant breeder is interested in generation mean analysis for $p$ correlated traits using information from a set of $m$ generations. Further assume that the generations permit the estimation of the components of means required. For instance, a set of four or more generations (e.g., $P_{1}, P_{2}, F_{1}, F_{2}, B_{1}, B_{2}$ ) may be raised to estimate the often needed components $m,[d]$, $[h]$ for a trait.

* ICRISAT Journal Article No. 294. 
If $Y_{k i j}$ is the measurement on the $k$ th trait of the $j$ th individual in $i$ th generation the associated genetic model is

$$
Y_{k i j}=x_{i}^{\prime} \beta_{k}+e_{k i j} \quad\left(k=1,2, \ldots p ; j=1,2, n_{i} ; i=1,2, \ldots m\right) .
$$

where $n_{i}$ is the number of individuals in $i$ th generation; $\boldsymbol{x}_{i}^{\prime}$ is a row vector of known constants and $\boldsymbol{\beta}_{k}$ is a vector of $q_{k}$ unknown parameters (components of means). For instance, if the generation observed is $B_{1}$ then for the three component model, $\boldsymbol{\beta}_{k}=(m,[d],[h]) ; q_{k}=3$ and $\boldsymbol{x}^{\prime}=(1,0.5,0.5)$. $e_{k i j}$ is assumed to be normally distributed with zero mean and $\sigma_{i k}^{2}$ variance. Covariance between $e_{k i j}$ and $e_{k^{\prime} i^{\prime} j^{\prime}}$ is $\sigma_{i k k^{\prime}}$ for $i=i^{\prime}, j=j^{\prime}$ and zero otherwise $\left(k \neq k^{\prime}\right)$.

Model (1) can be rewritten in terms of observed generation means:

$$
\bar{Y}_{k i}=x_{i}^{\prime} \beta_{k}+\bar{e}_{k i}
$$

or more compactly by

$$
\overline{\boldsymbol{Y}}_{k}=\boldsymbol{X}_{k} \boldsymbol{\beta}_{k}+\overline{\boldsymbol{e}}_{k}
$$

where $\bar{Y}_{k}=\left(\bar{Y}_{k 1}, \bar{Y}_{k 2}, \ldots, \bar{Y}_{k m}\right)^{\prime} ; \bar{e}_{k}=\left(\bar{e}_{k 1}, \bar{e}_{k 2}, \ldots, \bar{e}_{k m}\right)^{\prime}$;

$$
\boldsymbol{X}_{k}=\left[\begin{array}{c}
\boldsymbol{x}_{1}^{\prime} \\
\boldsymbol{x}_{2}^{\prime} \\
\vdots \\
\boldsymbol{x}_{m}^{\prime}
\end{array}\right] .
$$

The variance of the generation means for a trait $k$ can be written as

$$
E\left(\bar{e}_{k i}^{2}\right)=\sigma_{k i}^{2} / n_{i}
$$

and for the traits $k$ and $k^{\prime}$ the covariance between means of the same $i$ th generation as

and

$$
\operatorname{cov}\left(\bar{e}_{k i}, \bar{e}_{k^{\prime} i}\right)=\sigma_{i k k^{\prime}}^{\prime} / n_{i}
$$

$$
\operatorname{cov}\left(\bar{e}_{k i}, \bar{e}_{k^{\prime} i^{\prime}}\right)=0 \text { for } i \neq i^{\prime} .
$$

From the measurements made on the characters $k$ and $k^{\prime}$, the variance $\sigma_{i k}^{2}$ and covariance $\sigma_{i k k^{\prime}}$ within $i$ th generation can be estimated by

$$
\begin{gathered}
\hat{\sigma}_{i k}^{2}=\sum_{j=1}^{n_{i}}\left(Y_{k i j}-\bar{Y}_{k i}\right)^{2} /\left(n_{i}-1\right) \\
\hat{\sigma}_{i k k^{\prime}}=\sum_{j=1}^{n_{i}}\left(Y_{k i j}-\bar{Y}_{k i}\right)\left(Y_{k^{\prime} i j}-\bar{Y}_{k^{\prime} i}\right) /\left(n_{i}-1\right) .
\end{gathered}
$$

The model (2) for $k=1,2, \ldots, p$ becomes

$$
\boldsymbol{Y}=\boldsymbol{Z} \boldsymbol{\beta}+\boldsymbol{e}
$$

where

$$
\begin{gathered}
\boldsymbol{Y}=\left(\overline{\boldsymbol{Y}}_{1}^{\prime}, \ldots, \overline{\boldsymbol{Y}}_{p}^{\prime}\right)^{\prime} m p \times 1 \\
\boldsymbol{Z}=\left[\begin{array}{ccc}
\boldsymbol{X}_{1} & 0 & 0 \\
0 & \boldsymbol{X}_{2} & 0 \\
0 & \cdots & \boldsymbol{X}_{p}
\end{array}\right]_{m p \times q p} \quad \boldsymbol{\beta}=\left[\begin{array}{c}
\boldsymbol{\beta}_{1} \\
\vdots \\
\boldsymbol{\beta}_{p}
\end{array}\right]_{q p \times 1}
\end{gathered}
$$


When the same types of components of means are considered in all traits, then

and

$$
\boldsymbol{X}_{1}=\boldsymbol{X}_{2}=\cdots=\boldsymbol{X}_{p}=\boldsymbol{X}
$$

$$
\boldsymbol{Z}=\boldsymbol{I}_{p} \Theta \boldsymbol{X}
$$

where $I_{p}$ is an identity matrix of order $p$ and $\Theta$ represents Kronecker product of the two matrices involved.

$$
\boldsymbol{e}^{\prime}=\left(\overline{\boldsymbol{e}}_{1}^{\prime}, \ldots, \overline{\boldsymbol{e}}_{p}^{\prime}\right)
$$

is an $m p$-component row vector of normal variables with

and

$$
E(\boldsymbol{e})=\mathbf{0}
$$

$$
D(\boldsymbol{e})=\boldsymbol{W} .
$$

Where $E(\cdot)$ and $D(\cdot)$ represent the expectation vector and dispersion matrix of the random vector inside the operand. For $W$, we can write

$$
\boldsymbol{W}=\left[\begin{array}{ccc}
\boldsymbol{W}_{11} & \boldsymbol{W}_{12} & \boldsymbol{W}_{1 p} \\
\vdots & & \\
\boldsymbol{W}_{1 p} & \cdots & \boldsymbol{W}_{p p}
\end{array}\right]_{m p \times m p}
$$

where the $m \times m$ diagonal submatrices

$$
\boldsymbol{W}_{k k}=\operatorname{diag}\left(\sigma_{1 k}^{2} / n_{1}, \ldots, \sigma_{m k}^{2} / n_{m}\right)
$$

represent variance covariances of generation means for $k$ th trait and

$$
\boldsymbol{W}_{k k^{\prime}}=\operatorname{diag}\left(\sigma_{1 k k^{\prime}} / n_{1}, \ldots, \sigma_{m k k^{\prime}} / n_{m}\right)
$$

covariances between generation means of different $k$ and $k^{\prime}$ th traits.

Under the above model we shall consider the following.

\section{(i) Individual trait analysis}

For the $k$ th trait $(k=1,2, \ldots p)$ we have below the statistic $Q_{k}$ for testing the appropriateness of model (2) and the weighted least squares estimates $\hat{\boldsymbol{\beta}}_{k}$ of $\boldsymbol{\beta}_{k}$.

$$
\begin{aligned}
& Q_{k}=\left(\overline{\boldsymbol{Y}}_{k}-\boldsymbol{X}_{k} \hat{\boldsymbol{\beta}}_{k}\right)^{\prime} \boldsymbol{W}_{k k}^{-1}\left(\overline{\boldsymbol{Y}}_{k}-\boldsymbol{X}_{k} \hat{\boldsymbol{\beta}}_{k}\right) \\
& \hat{\boldsymbol{\beta}}_{k}=\left(\boldsymbol{X}^{\prime} \boldsymbol{W}_{k k}^{-1} \boldsymbol{X}_{k}\right)^{-1} \boldsymbol{X}_{k}^{\prime} \boldsymbol{W}_{k k}^{-1} \overline{\boldsymbol{Y}}_{k} .
\end{aligned}
$$

The distribution of $Q_{k}$ is approximately $\chi^{2}$ with $m-q_{k}$ degrees of freedom. It is approximate since $\boldsymbol{W}_{k k}$ contains estimated variances (Mather and Jinks 1971; Gale et al., 1977). The variance-covariance matrix of the estimated components of $\hat{\boldsymbol{\beta}}$ is given by

$$
D\left(\hat{\boldsymbol{\beta}}_{k}\right)=\left(\boldsymbol{X}_{k}^{\prime} \boldsymbol{W}_{k k}^{-1} \boldsymbol{X}_{k}\right)^{-1} .
$$

(ii) Several traits simultaneous analysis

We apply Aitken's (1935) generalised least squares analysis to obtain the statistic

$$
Q=(\boldsymbol{Y}-\boldsymbol{Z} \hat{\hat{\boldsymbol{\beta}}})^{\prime} \boldsymbol{W}^{-1}(\boldsymbol{Y}-\boldsymbol{Z} \hat{\hat{\boldsymbol{\beta}}})
$$


and test the appropriateness of the model (4) and $\hat{\hat{\beta}}$ as the estimator of $\beta$ where

$$
\hat{\boldsymbol{\beta}}=\left(\boldsymbol{Z}^{\prime} \boldsymbol{W}^{-1} \boldsymbol{Z}\right)^{-1} \boldsymbol{Z}^{\prime} \boldsymbol{W}^{-1} \boldsymbol{Y} \text {. }
$$

Writing $\boldsymbol{W}^{-1}$ as

$$
\boldsymbol{W}^{-1}=\left[\begin{array}{ccc}
\boldsymbol{W}^{11} & \cdots & \boldsymbol{W}^{1 p} \\
\vdots & & \\
\boldsymbol{W}^{p 1} & \cdots & \boldsymbol{W}^{p p}
\end{array}\right]
$$

we have

$$
\boldsymbol{Z}^{\prime} \boldsymbol{W}^{-1} \boldsymbol{Z}=\left[\begin{array}{ccc}
\boldsymbol{X}_{1}^{\prime} \boldsymbol{W}^{\prime \prime} \boldsymbol{X}_{1} & \cdots & \boldsymbol{X}_{1}^{\prime} \boldsymbol{W}^{1 p} \boldsymbol{X}_{p} \\
\vdots & & \\
\boldsymbol{X}_{p}^{\prime} \boldsymbol{W}^{p 1} \boldsymbol{X}_{1} & \cdots & \boldsymbol{X}_{p}^{\prime} \boldsymbol{W}^{p p} \boldsymbol{X}_{p}
\end{array}\right]
$$

and

$$
Z^{\prime} \boldsymbol{W}^{-1} Y=\left[\begin{array}{l}
\boldsymbol{X}_{1}^{\prime} \boldsymbol{W}^{11} \overline{\boldsymbol{Y}}_{1} \\
\vdots \\
\boldsymbol{X}_{p}^{\prime} \boldsymbol{W}^{1 p} \overline{\boldsymbol{Y}}_{p}
\end{array}\right]
$$

The estimator $\hat{\boldsymbol{\beta}}_{k}$ of $\boldsymbol{\beta}_{k}$ for the $k$-th trait is

$$
\hat{\hat{\boldsymbol{\beta}}}_{k}=\sum_{l=1}^{p} \boldsymbol{P}^{l k} \boldsymbol{X}_{1}^{\prime} \boldsymbol{W}^{l k} \boldsymbol{Y}_{k}
$$

where $\boldsymbol{p}^{i k}$ are $q_{l} \times q_{k}$ submatrices in the inverse of $\boldsymbol{Z}^{\prime} \boldsymbol{W}^{-1} \boldsymbol{Z}$ represented below

$$
\left(\boldsymbol{Z}^{\prime} \boldsymbol{W}^{-1} \boldsymbol{Z}\right)^{-1}=\left[\begin{array}{ccc}
\boldsymbol{P}^{11} & \cdots & \boldsymbol{p}^{1 p} \\
\vdots & & \\
\boldsymbol{P}^{p 1} & \cdots & \boldsymbol{P}^{p p}
\end{array}\right]
$$

The expression for $Q$ reduces to

$$
Q=\sum_{k} \sum_{k^{\prime}} \boldsymbol{r}_{k}^{\prime} \boldsymbol{W}^{k k^{\prime}} \boldsymbol{r}_{k^{\prime}}
$$

where residuals $\boldsymbol{r}_{k}=\overline{\boldsymbol{Y}}_{k}-\boldsymbol{X}_{k} \hat{\hat{\boldsymbol{\beta}}}_{\mathrm{k}}$.

The distribution of $Q$ is approximately $\chi^{2}$ with $m p=\sum_{k} q_{k}$ degrees of freedom. $Q$ simultaneously tests the appropriateness of model (4) for $p$ traits. The variances-covariances of estimates of various parameters are given by $D(\hat{\boldsymbol{\beta}})=\left(\boldsymbol{Z}^{\prime} \boldsymbol{W}^{-1} \boldsymbol{Z}\right)^{-1}$. Thus the variance-covariance matrices of the estimates of parameters of the same and different traits are

$$
\begin{gathered}
D\left(\hat{\hat{\boldsymbol{\beta}}}_{k}\right)=\boldsymbol{P}^{k k} \\
\operatorname{cov}\left(\hat{\hat{\boldsymbol{\beta}}}_{k}, \hat{\hat{\boldsymbol{\beta}}}_{k^{\prime}}\right)=\boldsymbol{P}^{k k^{\prime}} \quad k \neq k^{\prime}=1,2, \ldots, p .
\end{gathered}
$$

We can easily establish that the efficiency in sense of minimum variance of the two estimators $\hat{\boldsymbol{\beta}}_{k}$, using individual trait analysis, is less than or equal to that of $\hat{\boldsymbol{\beta}}_{k}$, using simultaneous analysis by the method of Rao (1973, p. 223 , ii). 


\section{The case of two traits}

This section illustrates the application of the simultaneous scaling test to the two traits situation. We also exhibit the dependence of the two tests based on $Q_{1}$ and $Q_{2}$ by working out the correlation between $Q_{1}$ and $Q_{2}$ for correlated traits.

For two traits situation,

$$
W=\left[\begin{array}{ll}
W_{11} & W_{12} \\
W_{12} & W_{22}
\end{array}\right] .
$$

To obtain the inverse of the matrices of type (5) we shall make use of Lemma 1 (Rao, 1973, p. 33).

Let $\boldsymbol{A}$ and $\boldsymbol{D}$ be two symmetric matrices such that inverses which occur in the expressions exist, then

$$
\left[\begin{array}{ll}
\boldsymbol{A} & \boldsymbol{B} \\
\boldsymbol{B}^{\prime} & \boldsymbol{D}
\end{array}\right]^{-1}=\left[\begin{array}{cc}
\boldsymbol{A}^{-1}+\boldsymbol{F} \boldsymbol{E}^{-1} \boldsymbol{F}^{\prime} & -\boldsymbol{F} \boldsymbol{E}^{-1} \\
-\boldsymbol{E}^{-1} \boldsymbol{F}^{\prime} & \boldsymbol{E}^{-1}
\end{array}\right]
$$

where

$$
\boldsymbol{E}=\boldsymbol{D}-\boldsymbol{B}^{\prime} \boldsymbol{A}^{-1} \boldsymbol{B} \text { and } \boldsymbol{F}=\boldsymbol{A}^{-1} \boldsymbol{B} .
$$

Thus, in

where

$$
\begin{aligned}
& W^{-1}=\left[\begin{array}{ll}
W^{11} & W^{12} \\
W^{12} & W^{22}
\end{array}\right] \\
& W^{11}=\operatorname{diag}\left(W_{111}, \ldots, W_{11 m}\right) \\
& W^{12}=\operatorname{diag}\left(W_{121}, \ldots, W_{12 m}\right) \\
& W^{22}=\operatorname{diag}\left(W_{221}, \ldots, W_{22 m}\right)
\end{aligned}
$$

$$
\begin{aligned}
W_{11 i} & =\sigma_{i 2}^{2} / \omega \\
W_{12 i} & =-\sigma_{i 12} / \omega \\
W_{22 i} & =\sigma_{i 1}^{2} / \omega \\
\omega & =\sigma_{i 1}^{2} \sigma_{i 2}^{2}-\sigma_{i 12}^{2} .
\end{aligned}
$$

Using Lemma 1, we can evaluate matrices $P$ 's in

$$
\left(Z^{\prime} W^{-1} Z\right)^{-1}=\left[\begin{array}{ll}
P^{11} & P^{12} \\
P^{21} & P^{22}
\end{array}\right]
$$

by

$$
\begin{aligned}
& P^{22}=\left(X_{2}^{\prime}\left(W^{22}-W^{12} X_{1}\left(X_{1}^{\prime} W^{11} X_{1}\right)^{-1} X_{1}^{\prime} W^{12}\right) X_{2}\right)^{-1} \\
& P^{12}=P^{211}=-\left(X_{1}^{\prime} W^{11} X_{1}\right)^{-1} X_{1}^{\prime} W^{12} X_{2} P^{22} \\
& P^{11}=\left(X_{1}^{\prime} W^{11} X_{1}\right)^{-1}\left(I_{q 1}+X_{1}^{\prime} W^{12} X_{2} P^{22} X_{2}^{\prime} W^{12} X_{1}\left(X_{1}^{\prime} W^{11} X_{1}\right)^{-1}\right)
\end{aligned}
$$

Furthermore

$$
\boldsymbol{Z}^{\prime} \boldsymbol{W}^{-1} \overline{\boldsymbol{Y}}=\left[\begin{array}{l}
U_{1} \\
U_{2}
\end{array}\right],
$$


where

$$
U_{1}=X_{1}^{\prime} W^{11} \bar{Y}_{1}+X_{1}^{\prime} W^{12} \bar{Y}_{2}, \quad U_{2}=X_{2}^{\prime} W^{12} \bar{Y}_{1}+X_{2}^{\prime} W^{22} \bar{Y}_{2}
$$

Therefore

$$
\hat{\hat{\boldsymbol{\beta}}}_{1}=\boldsymbol{P}^{11} \boldsymbol{U}_{1}+\boldsymbol{P}^{12} \boldsymbol{U}_{2}, \quad \hat{\hat{\boldsymbol{\beta}}}_{2}=\boldsymbol{P}^{21} \boldsymbol{U}_{1}+\boldsymbol{P}^{22} \boldsymbol{U}_{2} .
$$

For the statistic $Q$, we have

$$
\begin{aligned}
\boldsymbol{Q} & =(\overline{\boldsymbol{Y}}-\boldsymbol{Z} \hat{\hat{\boldsymbol{\beta}}})^{\prime} \boldsymbol{W}^{-1}(\overline{\boldsymbol{Y}}-\boldsymbol{Z} \hat{\hat{\boldsymbol{\beta}}}) \\
& =\overline{\boldsymbol{Y}}_{1}^{\prime} \boldsymbol{W}^{11} \overline{\boldsymbol{Y}}_{1}+2 \overline{\boldsymbol{Y}}_{1}^{\prime} \boldsymbol{W}^{12} \overline{\boldsymbol{Y}}_{2}+\boldsymbol{Y}_{2}^{\prime} \boldsymbol{W}^{22} \overline{\boldsymbol{Y}}_{2}-\hat{\hat{\boldsymbol{\beta}}}_{1}^{\prime} \boldsymbol{U}_{1}-\hat{\hat{\boldsymbol{\beta}}}_{2}^{\prime} \boldsymbol{U}_{2} \\
& =\boldsymbol{r}_{1}^{\prime} \boldsymbol{W}^{11} \boldsymbol{r}_{1}+2 \boldsymbol{r}_{1}^{\prime} \boldsymbol{W}^{12} \boldsymbol{r}_{2}+\boldsymbol{r}_{2}^{\prime} \boldsymbol{W}^{22} \boldsymbol{r}_{2}
\end{aligned}
$$

Here $\boldsymbol{r}_{1}$ and $\boldsymbol{r}_{2}$ are residual vectors obtained by fitting model (4).

Now, consider the analysis done on the individual traits and we have

with

$$
Q_{k}=\left(\overline{\boldsymbol{Y}}_{k}-\boldsymbol{X}_{k} \hat{\boldsymbol{\beta}}_{k}\right)^{\prime} \boldsymbol{W}_{k k}^{-1}\left(\overline{\boldsymbol{Y}}_{k}-\boldsymbol{X}_{k} \hat{\boldsymbol{\beta}}_{k}\right)
$$

$$
\hat{\boldsymbol{\beta}}_{k}=\left(\boldsymbol{X}_{k}^{\prime} \boldsymbol{W}_{k k}^{-1} \boldsymbol{X}_{k}\right)^{-1} \boldsymbol{X}_{k}^{\prime} \boldsymbol{W}_{k k}^{-1} \overline{\boldsymbol{Y}}_{k} \text {. }
$$

In order to evaluate the covariance between $Q_{1}$ and $Q_{2}$ we shall need Lemma 2 (Anderson, 1971, pp. 67, 73).

Let $\sum_{i j} a_{i j} U_{i} U_{j}=\boldsymbol{U}^{\prime} \boldsymbol{A} \boldsymbol{U}$, where $\boldsymbol{A}$ is symmetric and $E\left(U_{i}\right)=0, E\left(U_{i} U_{j}\right)=$ $0, i \neq j, E\left(U_{i}^{4}\right)=k_{4}+3 \sigma^{4}, E\left(U_{i}^{2} U_{j}^{2}\right)=\sigma^{4}, i \neq j$, and $E\left(U_{i} U_{j} U_{k} U_{l}\right)=0$ unless subscripts are equal in pairs. Then the mean and variance of the quadratic form $\boldsymbol{U}^{\prime} \boldsymbol{A} \boldsymbol{U}$ are

$$
\begin{aligned}
E\left(\boldsymbol{U}^{\prime} \boldsymbol{A} \boldsymbol{U}\right) & =\sigma^{2} \operatorname{tr} \boldsymbol{A} \\
\operatorname{Var}\left(\boldsymbol{U}^{\prime} \boldsymbol{A} \boldsymbol{U}\right) & =k_{4} \sum a_{i i}^{2}+2 \sigma^{4} \operatorname{tr} \boldsymbol{A}^{2} .
\end{aligned}
$$

The covariance between two quadratic forms $\sum_{i j} a_{i j} U_{i} U_{j}=\boldsymbol{U}^{\prime} \boldsymbol{A} \boldsymbol{U}$ and $\sum_{i j} b_{i j} U_{i} U_{j}=\boldsymbol{U}^{\prime} \boldsymbol{B U}$ is

$$
\operatorname{cov}\left(\boldsymbol{U}^{\prime} \boldsymbol{A} \boldsymbol{U}, \boldsymbol{U}^{\prime} \boldsymbol{B} \boldsymbol{U}\right)=k_{4} \sum a_{i i} b_{i i}+2 \sigma^{4} \operatorname{tr}(\boldsymbol{A B})
$$

where tr stands for the trace of the matrix.

With a little algebra on the matrices we can write

$$
\begin{aligned}
& Q_{1}=\boldsymbol{U}^{\prime} \boldsymbol{B}_{1} \boldsymbol{U} \\
& Q_{2}=\boldsymbol{U}^{\prime} \boldsymbol{B}_{2} \boldsymbol{U}
\end{aligned}
$$

where

$$
\begin{array}{rlr}
\boldsymbol{U} & =\boldsymbol{\Omega}^{-1} \boldsymbol{\varepsilon}, & \boldsymbol{\varepsilon}=\left(\boldsymbol{e}_{1}^{\prime}, \boldsymbol{e}_{2}^{\prime}\right)^{\prime} \\
\boldsymbol{B}_{k} & =\boldsymbol{\Omega} \boldsymbol{H}_{k} \boldsymbol{\Omega} & \\
\boldsymbol{\Omega} & =\boldsymbol{W}^{1 / 2} \\
\boldsymbol{H}_{\mathrm{l}} & =\left[\begin{array}{cc}
\boldsymbol{A}_{1} & 0 \\
0 & 0
\end{array}\right], \quad \boldsymbol{H}_{2}=\left[\begin{array}{cc}
0 & 0 \\
0 & \boldsymbol{A}_{2}
\end{array}\right] \\
\boldsymbol{A}_{\boldsymbol{k}} & =\left(\boldsymbol{I}_{m}-\boldsymbol{W}_{k k}^{-1} \boldsymbol{X}_{k}\left(\boldsymbol{X}_{k}^{\prime} \boldsymbol{W}_{k k}^{-1} \boldsymbol{X}_{k}\right)^{-1} \boldsymbol{X}_{k}\right) \boldsymbol{W}_{k k}^{-1} .
\end{array}
$$

Also note that $\boldsymbol{A}_{k} \boldsymbol{X}_{k}=\mathbf{0}$,

$$
\operatorname{tr}\left(\boldsymbol{A}_{k} \boldsymbol{W}_{k k}\right)=m-q_{k}, \quad k=1,2 .
$$


By writing

$$
\boldsymbol{\Omega}=\left[\begin{array}{ll}
\boldsymbol{\Omega}_{11} & \boldsymbol{\Omega}_{12} \\
\boldsymbol{\Omega}_{21} & \boldsymbol{\Omega}_{22}
\end{array}\right]
$$

we can further show that (6) satisfies the assumptions of Lemma 2 with

$$
\begin{aligned}
B_{1} & =\left[\begin{array}{ll}
\boldsymbol{\Omega}_{11} A_{1} \boldsymbol{\Omega}_{11} & \boldsymbol{\Omega}_{11} A_{1} \boldsymbol{\Omega}_{12} \\
\boldsymbol{\Omega}_{21} A_{1} \boldsymbol{\Omega}_{11} & \boldsymbol{\Omega}_{12} A_{1} \boldsymbol{\Omega}_{12}
\end{array}\right] \\
\boldsymbol{B}_{2} & =\left[\begin{array}{ll}
\boldsymbol{\Omega}_{12} A_{2} \boldsymbol{\Omega}_{21} & \boldsymbol{\Omega}_{12} A_{2} \boldsymbol{\Omega}_{22} \\
\boldsymbol{\Omega}_{21} \boldsymbol{A}_{2} \boldsymbol{\Omega}_{21} & \boldsymbol{\Omega}_{22} \boldsymbol{A}_{2} \boldsymbol{\Omega}_{22}
\end{array}\right] .
\end{aligned}
$$

Under the assumption that random vector $\varepsilon$ has multivariate normal distribution ( $k_{4}=0$, in Lemma 2 ) the variances of and covariance between the quadratic forms $Q_{1}$ and $Q_{2}$ are below (when $W$ is assumed to have true population values of variances and covariances).

$$
\begin{aligned}
E\left(Q_{k}\right) & =\operatorname{tr} \boldsymbol{B}_{k} \\
& =m-q_{k} \\
\operatorname{var}\left(Q_{k}\right) & =2 \operatorname{tr} \boldsymbol{B}_{k}^{2} \\
& =2\left(m-q_{k}\right)
\end{aligned}
$$

and

$$
\operatorname{cov}\left(Q_{1}, Q_{2}\right)=2 \operatorname{tr} B_{1} B_{2}
$$

We further note that

$$
\begin{aligned}
\operatorname{tr} B_{1} B_{2}= & \operatorname{tr}\left(\boldsymbol{\Omega}_{11} A_{1} \boldsymbol{\Omega}_{11} \boldsymbol{\Omega}_{12} A_{2} \boldsymbol{\Omega}_{21}+\boldsymbol{\Omega}_{11} A_{1} \boldsymbol{\Omega}_{12} \boldsymbol{\Omega}_{22} A_{2} \boldsymbol{\Omega}_{21}\right. \\
& \left.+\boldsymbol{\Omega}_{21} A_{1} \boldsymbol{\Omega}_{11} \boldsymbol{\Omega}_{12} A_{2} \boldsymbol{\Omega}_{22}+\boldsymbol{\Omega}_{21} A_{1} \boldsymbol{\Omega}_{12} \boldsymbol{\Omega}_{22} A_{2} \boldsymbol{\Omega}_{22}\right) \\
= & \operatorname{tr}\left(A_{1} W_{12} A_{2} W_{21}\right)
\end{aligned}
$$

using the relations

$$
\boldsymbol{\Omega}_{11} \boldsymbol{\Omega}_{12}+\boldsymbol{\Omega}_{12} \boldsymbol{\Omega}_{22}=\boldsymbol{W}_{12} .
$$

Further in terms of $\boldsymbol{W}$ 's and $\boldsymbol{X}$ 's we have

$$
\begin{aligned}
\operatorname{tr} B_{1} B_{2}= & \operatorname{tr}\left(\boldsymbol{W}_{11}^{-1} \boldsymbol{W}_{12} \boldsymbol{W}_{22}^{-1} \boldsymbol{W}_{21}-\left(\boldsymbol{X}_{1}^{\prime} \boldsymbol{W}_{11}^{-1} \boldsymbol{X}_{1}\right)^{-1} \boldsymbol{X}_{1}^{\prime} \boldsymbol{W}_{11}^{-1} \boldsymbol{W}_{12} \boldsymbol{W}_{22}^{-1} \boldsymbol{W}_{21} \boldsymbol{W}_{11}^{-1} \boldsymbol{X}_{1}\right. \\
& -\left(\boldsymbol{X}_{2}^{\prime} \boldsymbol{W}_{22}^{-1} \boldsymbol{X}_{2}\right)^{-1} \boldsymbol{X}_{2}^{\prime} \boldsymbol{W}_{22}^{-1} \boldsymbol{W}_{21} \boldsymbol{W}_{11}^{-1} \boldsymbol{W}_{12} \boldsymbol{W}_{22}^{-1} \boldsymbol{X}_{2} \\
& +\left(\boldsymbol{X}_{1}^{\prime} \boldsymbol{W}_{11}^{-1} \boldsymbol{X}_{1}\right)^{-1} \boldsymbol{X}_{1}^{\prime} \boldsymbol{W}_{11}^{-1} \boldsymbol{W}_{12} \boldsymbol{W}_{22}^{-1} \boldsymbol{X}_{2} \\
& \left.\times\left(\boldsymbol{X}_{2}^{\prime} \boldsymbol{W}_{22}^{-1} \boldsymbol{X}_{2}\right)^{-1} \boldsymbol{X}_{2}^{\prime} \boldsymbol{W}_{22}^{-1} \boldsymbol{W}_{22} \boldsymbol{W}_{11}^{-1} \boldsymbol{X}_{1}\right)
\end{aligned}
$$

The expression (7) of covariance between $Q_{1}$ and $Q_{2}$ reduces to zero when the two traits are uncorrelated i.e., $W_{12}=0$. Thus the tests based on $Q_{1}$ and $Q_{2}$ are dependent in general for testing the genetic model for two correlated traits.

\section{ILLUSTRATION}

An application of the method discussed in this paper has been made by the referee and his colleague on their data (details of the data available 
on request from Dr H.S. Pooni) and is produced in the following with their consent. The data were obtained on four traits $\left(\mathrm{H}_{1}, \mathrm{H}_{2}\right.$ : juvenile height, measured on two occasions two weeks apart; $\mathrm{H}_{\mathrm{F}}$ : height at flowering and $\mathrm{L}_{\mathbf{s}}$ : leaf spread) in Nicotiana rustica. The total number of plants raised was 500.

Table 1 presents the correlation coefficients for the pairs of traits used in analysis.

TABLE 1

Correlation coefficients between traits observed for various generations

\begin{tabular}{ccccc}
\hline Generations & $\mathrm{H}_{1}, \mathrm{H}_{2}$ & $\mathrm{H}_{1}, \mathrm{H}_{\mathrm{F}}$ & $\mathrm{H}_{l}, \mathrm{~L}_{\mathrm{S}}$ & $\mathrm{H}_{\mathrm{F}}, \mathrm{H}_{\mathrm{S}}$ \\
\hline $\mathrm{P}_{1}$ & 0.96 & -0.17 & -0.44 & 0.66 \\
$\mathrm{P}_{2}$ & 0.86 & -0.48 & -0.28 & 0.76 \\
$\mathrm{~F}_{1}$ & 0.93 & -0.35 & -0.26 & 0.70 \\
$\mathrm{~F}_{2}$ & 0.94 & -0.38 & -0.37 & 0.52 \\
$\mathrm{~B}_{1}$ & 0.93 & -0.48 & -0.42 & 0.66 \\
$\mathrm{~B}_{2}$ & 0.88 & -0.60 & -0.33 & 0.56 \\
\hline
\end{tabular}

The estimates of the parameters $m,[d],[h]$ for the two highly correlated traits $\mathrm{H}_{1}$ and $\mathrm{H}_{2}$ are given in table 2 using individual traits and simultaneous traits analyses. The variances of the estimates are given in parentheses.

\section{TABLE 2}

Estimates of parameters $m,[d],[h]$ and their variances for traits $H_{1}$ and $H_{2}$ Traits and estimation method

\begin{tabular}{cccrr}
\hline & \multicolumn{2}{c}{$\mathrm{H}_{1}$} & \multicolumn{2}{c}{$\mathrm{H}_{2}$} \\
\cline { 2 - 5 } Parameters & Individual & Simultaneous & Individual & Simultaneous \\
\hline$m$ & $5.341(0.027)$ & $5.346(0.027)$ & $15.776(0.255)$ & $15.764(0.254)$ \\
{$[d]$} & $1.090(0.025)$ & $1.101(0.025)$ & $3.388(0.235)$ & $3.374(0.235)$ \\
{$[h]$} & $0.386(0.095)$ & $0.349(0.094)$ & $1 \cdot 314(0.890)$ & $1 \cdot 323(0.888)$ \\
\hline
\end{tabular}

In table 2 simultaneous method means that $\mathrm{H}_{1}$ and $\mathrm{H}_{2}$, but no other characters, were analysed simultaneously.

We now consider the estimation of parameters for three traits $\mathrm{H}_{1}, \mathrm{H}_{\mathrm{F}}$ and $\mathrm{L}_{\mathrm{S}}$ analysed individually and according to schemes

(a) $\mathrm{H}_{1}, \mathrm{H}_{\mathrm{F}}$ simultaneously

(b) $\mathrm{H}_{\mathrm{I}}, \mathrm{L}_{\mathrm{S}}$ simultaneously

(c) $\mathrm{H}_{1}, \mathrm{H}_{\mathrm{F}}$ and $\mathrm{L}_{\mathrm{S}}$ simultaneously

From the comparison of the variances of the estimates of the various parameters resulting from individual and simultaneous traits analyses in table 2 and table 3, we notice that there is improvement in efficiency by inclusion of two or more traits in analyses. However, this improvement is relatively small. The improvement in efficiency will be determined by the degree of correlation between the traits over these generations. It is expected that improvement in efficiency will be greatest when correlations are intermediate. When all correlations are zero then simultaneous traits analysis 
TABLE 3

Estimates of parameters $m,[d],[h]$ and their variances for traits $H_{1}, H_{F}$ and $L_{S}$

\begin{tabular}{|c|c|c|c|c|c|}
\hline \multirow[b]{2}{*}{ Traits } & \multirow[b]{2}{*}{ Parameter } & \multicolumn{4}{|c|}{ Estimation method } \\
\hline & & Individual & Scheme (a) & Scheme (b) & Scheme (c) \\
\hline $\mathbf{H}_{1}$ & $\begin{array}{c}m \\
{[d]} \\
{[h]}\end{array}$ & $\begin{array}{l}5.341(0.027) \\
1.090(0.027) \\
0.386(0.095)\end{array}$ & $\begin{array}{l}5.330(0.026) \\
1.094(0.024) \\
0.462(0.091)\end{array}$ & $\begin{array}{l}5.335(0.027) \\
1.087(0.025) \\
0.417(0.094)\end{array}$ & $\begin{array}{l}5.341(0.026) \\
1.099(0.023) \\
0.421(0.089)\end{array}$ \\
\hline $\mathbf{H}_{\mathrm{F}}$ & $\begin{array}{c}m \\
{[d]} \\
{[h]}\end{array}$ & $\begin{array}{r}67.813(0.682) \\
-5.896(0.666) \\
6.137(0.457)\end{array}$ & $\begin{array}{r}67.789(0.681) \\
-5.869(0.663) \\
6.176(0.447)\end{array}$ & & $\begin{array}{r}67.836(0.678) \\
-5.985(0.654) \\
6.395(0.429)\end{array}$ \\
\hline $\mathrm{L}_{\mathrm{s}}$ & $\begin{array}{l}\mathrm{m} \\
{[d]} \\
{[h]}\end{array}$ & $\begin{array}{r}42.762(0.240) \\
-0.383(0.223) \\
6.585(0.894)\end{array}$ & & $\begin{array}{r}42.747(0.239) \\
-0.379(0.222) \\
6.608(0.888)\end{array}$ & $\begin{array}{r}42.749(0.234) \\
-0.418(0.219) \\
6.628(0.863)\end{array}$ \\
\hline
\end{tabular}

reduces to the individual trait analyses. This procedure breaks down if any of the correlations is unity. An empirical study can be made to examine the performance of the estimates obtained by the two methods by taking several values for correlation coefficients for generations in its admissible range. (Also see section 2(ii).)

Acknowledgements. The author is thankful to Professor J. L. Jinks, Dr H. S. Pooni and Dr C. S. Haley, Department of Genetics, University of Birmingham for encouraging comments and providing the analysis carried out on their data as reported in section 4 . I also thank $\mathrm{Mr}$ B. Gilliver for his encouragement in this work and Mr C. P. Jaiswal for typing the manuscript.

\section{REFERENCES}

ANDERSON, T. W. 1971. The Statistical Analysis of Time Series. John Wiley and Sons Inc., New York.

AITKEN, A. C. 1935. On least squares and linear combinations of observations. Proc. Roy. Soc. Edin. A, 55, 42-48.

CAVALLI, L. L. 1952. An analysis of linkage in quantitative inheritance. In Reeve, E. C. R. and Waddington, C. H. (ed.) Quantitative Inheritance, H.M.S.O., London.

DWIVEDI. S. L. 1978. Generation mean analysis of quantitative characters in rice. Personal communication, ICRISAT, Patancheru, A.P., India.

GALE, J. G., MATHER, K. AND JINKS, J. L. 1977. Joint scaling tests. Heredity, 38, 47-51.

LAW, C. N., SNAPE, J. W. AND WORLAND, A. J. 1978. The genetical relationship between height and yield in wheat. Heredity, 40, 133-151.

MATHER, K. AND JINKS, J. L. 1971. Biometrical Genetics. Chapman and Hall, London.

PATHAK, R. S. AND SINGH, R. B. 1970. Genetics of yield characters in upland cotton. Indian Journal of Genetics and Plant Breeding, 30, 679-689.

RAO, C. R. 1973. Linear Statistical Inference and its Application. John Wiley, New York. 